\title{
3-D model for cosmic ray planetary ionisation in the middle atmosphere
}

\author{
P. I. Y. Velinov ${ }^{1}$, L. Mateev ${ }^{1}$, and N. Kilifarska ${ }^{2}$ \\ ${ }^{1}$ Central Solar-Terrestrial Influences Laboratory, Academician Dimitar Mishev, Bulgarian Academy of Sciences, Acad. G. \\ Bonchev Str. Bl.3, 1113, Sofia, Bulgaria \\ ${ }^{2}$ Geophysical Institute, Bulgarian Academy of Sciences, Acad. G. Bonchev Str. Bl. 3, 1113 Sofia, Bulgaria
}

Received: 22 February 2005 - Revised: 1 July 2005 - Accepted: 20 July 2005 - Published: 22 November 2005

Part of Special Issue "1st European Space Weather Week (ESWW)"

\begin{abstract}
A 3-D planetary model of the cosmic ray electron production rate $\mathrm{q}(\mathrm{h})\left(\mathrm{cm}^{-3} \mathrm{~s}^{-1}\right)$ has been developed for the strato-mesosphere and lower thermosphere (altitude range $30-100 \mathrm{~km}$ ) with a $10-\mathrm{km}$ step. The spectrum of the primary cosmic rays is modelled by an analytical expression using input data from the CREME96 model. An isotropic penetration of the cosmic rays from the upper hemisphere is assumed in the model and a spherical shape of the Earth's atmosphere is taken into account. The longitudinal effect of ionisation from cosmic rays in the mesosphere and lower thermosphere is calculated. A graphical presentation of the computational results is given for 50 and $90 \mathrm{~km}$ at $0^{\circ}, 40^{\circ}, 50^{\circ}$ and $70^{\circ} \mathrm{N}, \mathrm{S}$ latitudes and all longitudes with a step of $30^{\circ}$. The results of this paper provide a basis for a quantitative understanding of the energetic processes of the middle atmosphere and mechanisms affecting the thermodynamical balance of the Earth's atmosphere.
\end{abstract}

Keywords. Ionosphere (Solar radiation and cosmic ray effects; Ionisation mechanisms; Ionosphere-atmosphere interactions)

\section{Introduction}

The galactic cosmic rays create the lower part of the terrestrial ionosphere. They form an independent cosmic ray (CR) layer in the planetary ionosphere, the so-called CR layer in the ionospheric D-region, which is situated at altitudes 50$80 \mathrm{~km}$. Modern data show dominance of Lyman-alpha ionisation at $75-80 \mathrm{~km}$, except for $\phi=70^{\circ}$ (Velinov, 1966; 1968). In this way the galactic cosmic rays influence the propagation of medium, long and very long radio waves (Velinov et al., 1974). The cosmic rays maintain the ionisation not only

Correspondence to: P. I. Y. Velinov

(pvelinov@bas.bg) in the lower ionosphere, but also in the atmosphere, the hydrosphere and the lithosphere of the Earth (Dorman, 2004).

The cosmic rays determine the electron production rates, the electron densities and electrical conductivities in the middle (below about $70 \mathrm{~km}$ ) and lower atmosphere, and therefore the atmospheric electric fields (Tonev and Velinov, 2002; Dorman, 2004), which influence the global electric circuit between the ionosphere and the ground, the Earth's global charge and the thunderstorms. It is already established that the main cause of thunderstorm discharges are external atmospheric showers (EAS) of high energy primary CR particles with energies of more than $10^{14} \mathrm{eV}$ (Dorman, 2004).

The goal of the present paper is to give new and improved results for the model of ionisation by galactic cosmic rays at the altitudes $30-100 \mathrm{~km}$. Some previous results (Velinov and Mateev, 1993; Velinov et al., 1974) will be specified and generalised in this way.

Being a transition region between the upper atmosphere (fully solar controlled) and the lower levels (meteorologically controlled), the lower ionosphere, the so-called Dregion, exhibits both solar and meteorological control. There is a tendency of the spatial variability in the low frequency (LF) radio-wave absorption to be attributed to the longitudinal variations in the dynamical regime of the mesosphere and lower thermosphere (Kazimirovsky et al., 2003). The charged particles in a weakly ionised lower ionosphere are really controlled by neutral wind dynamics. However, some short lasting local enhancements in LF radio-wave absorption may have their solar or galactic origin.

\section{Three-dimensional ionisation model}

Velinov et al. (2001, 2004) developed a model of the high-energy particle ionisation in the middle and upper atmosphere. Formulas for the electron production rate $\mathrm{q}\left(\mathrm{cm}^{-3} \mathrm{~s}^{-1}\right)$ of high-energy particles penetrating into a 


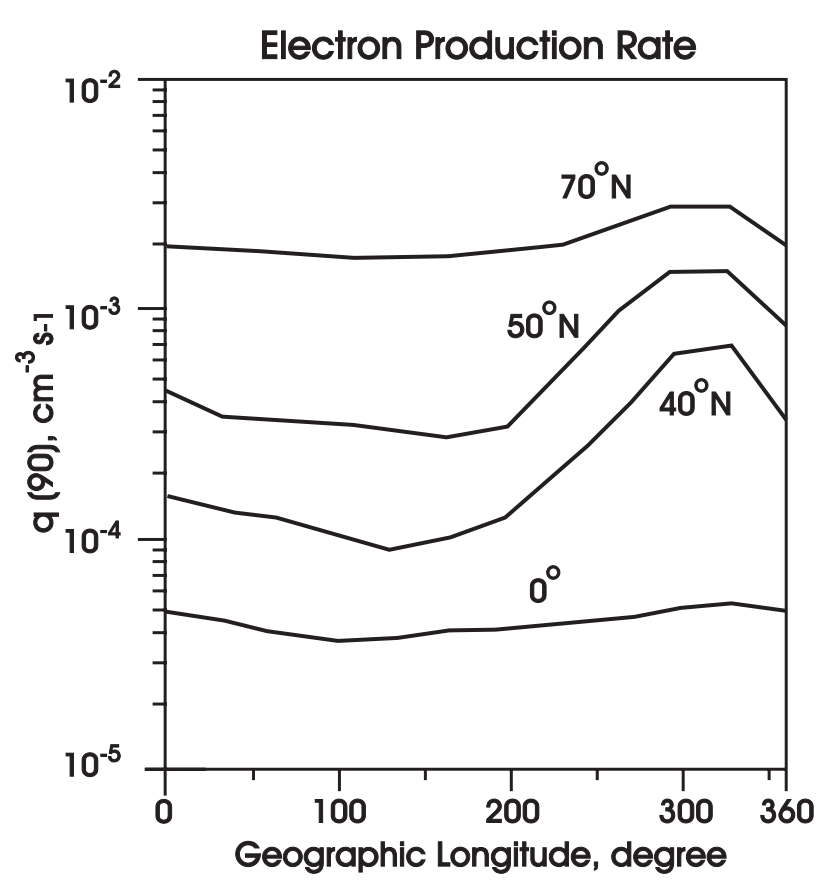

Fig. 1. Planetary distribution of electron production rate by galactic cosmic rays at the height of $90 \mathrm{~km}$ in the Northern Hemisphere.

planetary ionosphere have been deduced for galactic (Velinov, 1966) and solar (Velinov, 1968) cosmic rays. The electron production rate $q$ as a function of height $h(\mathrm{~km})$ for given particles of type $i$ (from the cosmic ray composition) can be written as follows (Velinov et al., 1974; Velinov, 1966, 1968):

$$
\begin{aligned}
& q(h)=\sum_{i} q_{i}(h)= \\
& \frac{1}{Q} \sum_{i} \int_{E_{i}}^{\infty} \int_{A=0}^{2 \pi} \int_{\theta=0}^{\pi / 2+\Delta \theta} D_{i}(E)\left(\frac{d E}{d h}\right)_{i} \sin \theta d \theta d A d E
\end{aligned}
$$

where $Q$ is the energy required for the formation of one electron-ion pair and depends on the atmospheric composition; $D_{i}(E)$ is the differential spectrum of the particles; $E$ is their kinetic energy; $E_{i}$ is the energy $(\mathrm{GeV} / \mathrm{nucl})$, which corresponds to the geomagnetic cut-off rigidity $R_{C}(\mathrm{GV}) ; d E / d h$ represents the ionisation loss of the penetrating cosmic rays (CR) particles, expressed by the Bohr-Bethe-Bloch formula; $A$ is the azimuth angle; $\theta$ is the angle towards the vertical; $\Delta \theta$ takes into account that at a given height the particles can penetrate from the space angle $\left(0^{\circ}, \theta_{\max }=90^{\circ}+\Delta \theta\right)$, which is greater than the upper hemisphere angle $\left(0^{\circ}, 90^{\circ}\right)$ for a flat model. Out of the elementary geometric considerations the following can be derived for spherical model (Velinov, 1968):

$\Delta \theta=\pi / 2-\arccos \left(\left(2 a h+h^{2}\right)^{1 / 2} /(a+h)\right)$, where $a$ is the planetary radius. For the heights $30-80 \mathrm{~km}$ in the terrestrial atmosphere $\Delta \theta$ varies from $6^{\circ}$ to $9^{\circ}$, i.e. $\theta_{\max }$ varies from $96^{\circ}$ to $99^{\circ}$.

The summation in the ionisation integral Eq. (1) is made on the groups of nuclei: protons $p$, Helium ( $\alpha$-particles), light $L(3 \leq Z \leq 5)$, medium $M(6 \leq Z \leq 9)$, heavy $H(Z \geq 10)$ and very heavy $\mathrm{VH}(Z \geq 20)$ nuclei in the composition of cosmic rays; $Z$ is the charge of the nuclei (Hillas, 1972; Webber, 1967). Detailed data for the energy loss $Q$ in producing a $(+,-)$ ion pair in different gases and solar-composition gas mixtures are given in Lewis (1997; 167-169). The ionisation efficiency in rare gases is somewhat higher (about 60\%) because the rare gases have no capacity for absorbing energy to excite vibration, rotation or dissociation; only ionisation and translational excitation are possible.

The mathematical method for solving this threedimensional integral is a Gaussian quadrature with Legendre polynomials for 10 root points (Press et al., 2002). The procedure uses the possibility in the Pascal algorithmic language for recursive calls of the integrand. This allows for the ionisation profiles to be evaluated with an account of the energetic CR spectra and their transformation along their way throughout the atmosphere. With the spherical shape of the atmosphere and the geomagnetically controled cut-off rigidity (the critical level of rigidity, i.e. momentum of particle divided on its charge, below which the particles are "bent" away of the atmosphere), $R_{C}$ is taken into account. The longitudinal effect that we seek appears as a result of the corresponding CR longitudinal effect (due to the displacements of geomagnetic and geographic poles).

\section{Results for planetary ionisation}

The longitudinal distributions of the ionisation rate (or electron production rate) with a step of $30^{\circ}$, at two different altitudes $(50$ and $90 \mathrm{~km})$, at $0^{\circ}, 40^{\circ}, 50^{\circ}$ and $70^{\circ}$ latitudes, are shown in Figs. 1, 3 for the Northern Hemisphere and Figs. 2, 4 for Southern Hemisphere, correspondingly. The atmosphere density profiles are taken from the model CIRA (1989).

The computations are made for minimum solar activity, when the intensity of the galactic cosmic rays is maximal. The spectrum of the primary cosmic rays is modelled with an analytical expression, with input data from the model CREME96 (2002). Our model takes into account the isotropic penetration of the cosmic rays from the upper hemisphere and the spherical shape of the Earth's atmosphere by means of the Chapman function.

From Figs. 1-4 the considerable longitudinal effect in the cosmic ray layer in the lower ionosphere and the respective planetary ionisation can be seen. The irregular distribution of the ionisation rate $q(h)$ is especially well pronounced at mid-latitudes. Despite the amplitudes of the longitudinal effect at both levels being comparable, the absolute value of $q(h)$ is much higher at $50 \mathrm{~km}$ than at $90 \mathrm{~km}$, due to the enhanced efficiency of $\mathrm{CR}$ with an increase in the atmospheric 


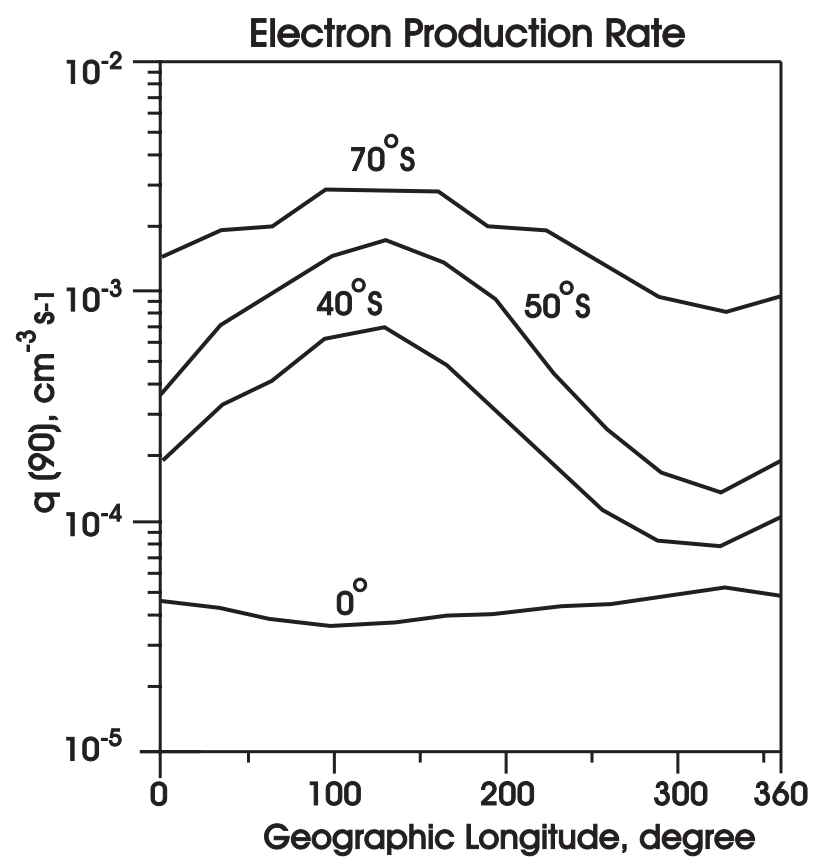

Fig. 2. Planetary distribution of electron production rate by galactic cosmic rays at the height of $90 \mathrm{~km}$ in the Southern Hemisphere.

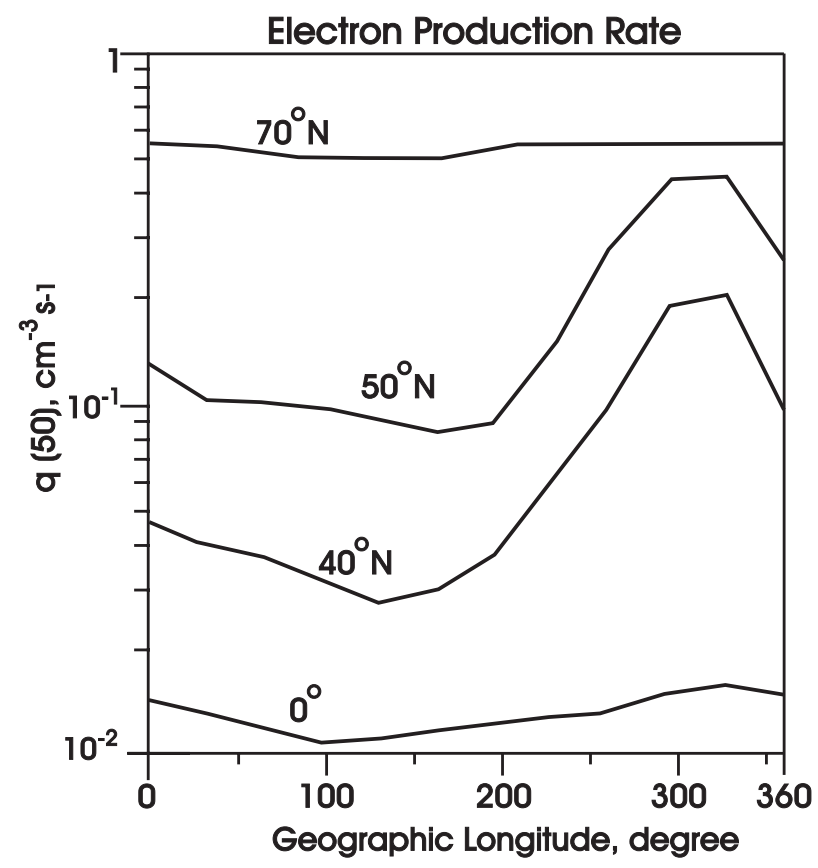

Fig. 3. Planetary distribution of electron production rate by galactic cosmic rays at the height of $50 \mathrm{~km}$ in the Northern Hemisphere.

optical depth. Unlike the F-region, where the spatial distribution of plasma density in the Northern Hemisphere is described by wave number 2 (two minima and two maximums), the longitudinal variability of the electron production rate in the lower ionosphere corresponds to wave number 1 in both

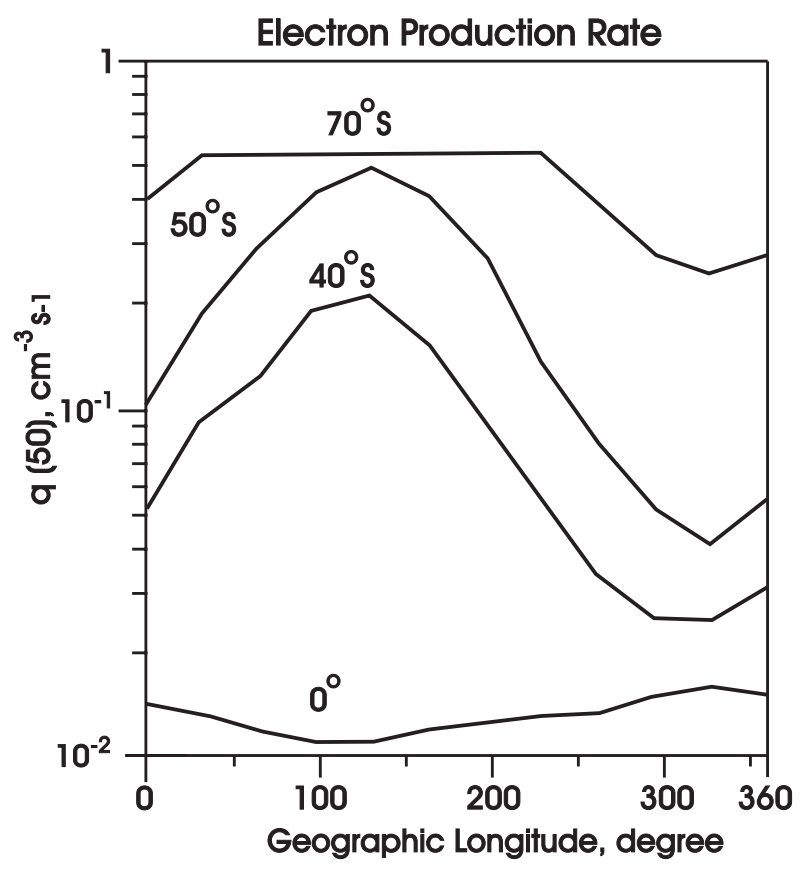

Fig. 4. Planetary distribution of electron production rate by galactic cosmic rays at the height of $50 \mathrm{~km}$ in the Southern Hemisphere.

Table 1. Ratio of maximal and minimal values of the electron production rate and the electron density for fixed geomagnetic latitudes $0^{\circ}, 40^{\circ}, 50^{\circ}$ and $70^{\circ}$.

\begin{tabular}{lcrrr}
\hline Latitude & $0^{\circ}$ & $40^{\circ}$ & $50^{\circ}$ & $70^{\circ}$ \\
\hline$q_{\max }(50) / q_{\min }(50)$ & 1.45 & $7.59 \mathrm{~N}$ & $5.36 \mathrm{~N}$ & $1.12 \mathrm{~N}$ \\
& & $8.72 \mathrm{~S}$ & $12.44 \mathrm{~S}$ & $2.26 \mathrm{~S}$ \\
$N_{\max }(50) / N_{\min }(50)$ & 1.2 & $2.75 \mathrm{~N}$ & $2.31 \mathrm{~N}$ & $1.06 \mathrm{~N}$ \\
& & $2.95 \mathrm{~S}$ & $3.53 \mathrm{~S}$ & $1.5 \mathrm{~S}$ \\
\hline
\end{tabular}

hemispheres. The main reason for this result is that charged energetic particles, penetrating the Earth's atmosphere are strongly controlled by the inclination (the slope) of the magnetic field lines. At mid-latitudes and longitudes $250^{\circ}-300^{\circ}$ in the Northern Hemisphere and $100^{\circ}-150^{\circ}$ in the Southern Hemisphere they enter the atmosphere at the highest angles (closest to the vertical) and penetrate deeper into the atmosphere, ionising neutral particles on their way. At these altitudes the primary ionisation is more effective and this explains the great differences (see Table 1) with longitudes where the CR penetrates at lower angles (regarding the horizon). In the upper ionosphere both the magnetic inclination and declination $(D)$ (i.e. the angle between horizontal component of magnetic field and north direction) affect the drift of the plasma and, respectively, its spatial distribution. At every map of magnetic field declination one can see that the spatial distribution of $D$ in the Northern Hemisphere (NH) is described by wave number 2. Correspondingly, observations and modelling revealed the existence of 2 minima and 
2 maxima in the $\mathrm{NH}$ upper ionosphere plasma distribution (Chalinor and Eccles, 1971; Kilifarska et al., 1991).

Table 1 presents the amplitudes of longitudinal effect in electron production rate $q$ and electron concentration $N$ at $50 \mathrm{~km}$, calculated by the expressions:

$N=\left(q / \alpha_{1}\right)^{1 / 2}, N_{\max } / N_{\min }=\left(q_{\max } / q_{\min }\right)^{1 / 2}$,

where $\alpha_{1}$ is the effective recombination coefficient.

The ratio of maximal and minimal values of the electron production rate and the electron density for fixed geomagnetic latitudes $0^{\circ}, 40^{\circ}, 50^{\circ}$ and $70^{\circ}$ is given in Table 1 . From Table 1 it is seen that the maximum amplitude of $q_{\max }(50) / q_{\min }(50)$ longitudinal variations is at mid-latitudes $40^{\circ}-50^{\circ}$. Our results are also in good agreement with ionisation rates calculated based on ground neutral monitor measurements (O'Brian and Sauer, 2001)

\section{Discussion and conclusions}

A 3-D planetary model of the cosmic ray electron production rate $\mathrm{q}(\mathrm{h})\left(\mathrm{cm}^{-3} \mathrm{~s}^{-1}\right)$ has been developed covering the altitude range $30-100 \mathrm{~km}$ (with $10 \mathrm{~km}$ step), latitudes $70^{\circ} \mathrm{S}-70^{\circ} \mathrm{N}$ and all longitudes with a step of $30^{\circ}$. In the high latitude and polar regions (especially for the latitudes $70^{\circ} \mathrm{N}$ and $70^{\circ} \mathrm{S}$ ) the contribution of the anomalous component of cosmic rays with energies less than $50-60 \mathrm{MeV}$ increase significantly. This component is also included in our model. The calculations are made for low solar activity, when the galactic cosmic ray fluxes have their maximum and do not account for the ionisation produced from solar energetic particles.

The longitudinal variability of ionisation rate production, revealed by the model, can be explained through spatial irregularities of the Earth's magnetic field, particularly magnetic inclination (i.e. the slope of the magnetic lines). At mid-latitude, where the longitudinal effect has maximum amplitude, there are two regions, respectively, at longitudes $250^{\circ}-300^{\circ}$ in the Northern Hemisphere and $100^{\circ}-150^{\circ}$ in the Southern Hemisphere, where the cosmic particles enter the atmosphere at the highest angles. The efficiency of the primary ionisation increases with enhancing the angle of entering the atmosphere, which explains the great amplitude of longitudinal variability in CR ionisation rates (the maximum concentration is twice - to triple the minimum one). The results in Figs. 1-4 relate to minimum solar activity, when the cosmic ray intensity is maximal. We will calculate in the future the cosmic ray planetary ionisation for the average and maximal solar activity. In this way we will account for the 11-year cosmic ray modulation by the solar wind. The next step in the development of this problem is the calculation of the electron density $N\left(\mathrm{~cm}^{-3}\right)$ created by cosmic rays. The values of $N$ can be obtained, if the planetary distribution of recombination coefficients is known. However, some difficulties arise in the consideration of this problem (Dorman, 2004).
Acknowledgements. Topical Editor T. Pulkkinen thanks L. I. Dorman and another referee for their help in evaluating this paper.

\section{References}

CIRA 1989: COSPAR International Reference Atmosphere, NorthHolland, Amsterdam, 1989.

Chalinor, R. and Eccles D.: Longitudinal variations of mid-latitude ionosphere produced by neutral-air winds, J. Atmos. Terr. Phys., 33, 363-369, 1971.

CREME96: Cosmic Ray Ion Differential and Integral Spectra (Last Modified: 6 August 2002). http://www.estec.esa.nl/wmwww/ wma/ProjectSupport/ISO/CREME96.html.

Dorman L. I.: Cosmic Rays in the Earth's Atmosphere and Underground, Kluwer Acad. Publ., Dordrecht, 2004.

Hillas, A. M.: Cosmic Rays, Pergamon Press, Oxford, 1972.

Kazimirovsky, E., Herraiz, M., and de la Morena, B. A.: Effects on the ionosphere due to phenomena occurring below it, Surv. Geophys., 24, 139-184, 2003.

Kilifarska, N., Dachev, T., Besprozvannaya, A., and Schuka, T.: An influence of IMF-By on the ion density planetary distribution during disturbed period 20-23 October 1981: satellite and ground-based results, Adv. Space Res., 11, 47-50, 1991.

Lewis, J. S.: Physics and Chemistry of the Solar System, Academic Press, San Diego, California, 1997.

O'Brian, K. and Sauer, H.: On the atmospheric response to solar particle events, Internat. J. Geomag. Aeron., 2, 181-188, 2001.

Press, W. H., Flannery, B. P., Teukolsky, S. A., and Vetterling, W. T.: Numerical Recipes in C - The Art of Scientific Computing, Cambridge University Press, Cambridge, 2002.

Tonev P. T. and Velinov, P. I. Y.: Electrostatic Fields above Thunderclouds at Different Latitudes and their Ionospheric Effects, Adv. Space Res., 30, 2625-2630, 2002.

Velinov, P. I. Y.: An Expression for Ionospheric Electron Production Rate by Cosmic Rays, Compt. rend., Acad. Bulg. Sci., 19, 109112, 1966.

Velinov, P. I. Y.: On Ionisation of the Ionospheric D-Region by Galactic and Solar Cosmic Rays, J. Atmos. Terr. Phys., 30, 18911905, 1968.

Velinov, P. I. Y., Buchvarova, M., Mateev, L., and Ruder, H.: Determination of Electron Production Rates Caused by Cosmic Ray Particles in Ionospheres of Terrestrial Planets, Adv. Space Res., 27, 1901-1908, 2001.

Velinov, P. I. Y. and Mateev, L. N.: Three-Dimensional Global Modelling of the Middle Atmosphere Ionisation and its Relation to Longitudinal Effects, Adv. Space Res., 13, 377-380, 1993.

Velinov, P. I. Y., Nestorov, G., and Dorman, L. I.: Cosmic Ray Effects on the Ionosphere and on the Radiowaves Propagation, Publ. House of Bulg. Acad. Sci., Sofia, 1974.

Velinov, P. I. Y., Ruder, H., Kostov, V., Mateev, L. N., and Buchvarova, M.: Method for Calculation of Ionisation Profiles Caused by Cosmic Rays in Giant Planet Ionospheres from Jovian Group, Adv. Space Res., 33, 232-239, 2004.

Webber, W. R.: Cosmic Ray, in: Handbuch der Physik, XLVI/2, Springer Verlag, Berlin, 1967. 